\title{
HOUSEHOLDER'S APPROXIMANTS AND CONTINUED FRACTION EXPANSION OF QUADRATIC IRRATIONALS
}

\author{
VINKO PETRIČEvić \\ University of Zagreb, Croatia
}

\begin{abstract}
There are numerous methods for rational approximation of real numbers. Continued fraction convergent is one of them and Newton's iterative method is another one. Connections between these two approximation methods were discussed by several authors. Householder's methods are generalisation of Newton's method. In this paper, we will show that for these methods analogous connection with continued fractions hold.
\end{abstract}

\section{INTRODUCTION AND MAIN RESUlts}

Let $\alpha$ be a quadratic irrational, i.e., $\alpha=c+\sqrt{d}, c, d \in \mathbb{Q}, d>0$ and $d$ is not a square of a rational number. It is well known that continued fraction expansion of $\alpha$ is periodic, i.e., has the form

$$
\alpha=\left[a_{0}, a_{1}, \ldots, a_{h}, \overline{a_{h+1}, a_{h+2}, \ldots, a_{h+\ell}}\right] .
$$

Here $\ell=\ell(\alpha)$ denotes the length of the shortest period in the expansion of $\alpha$. We will observe quadratic irrationals whose period begins with $a_{1}$. We will say that period is palindromic if it holds $a_{1}=a_{\ell-1}, a_{2}=a_{\ell-2}, \ldots$, i.e., the period without the last term is symmetric.

Continued fraction convergents $\frac{p_{n}}{q_{n}}=\left[a_{0}, a_{1}, \ldots, a_{n}\right]$ give good rational approximations of $\alpha$. Another approximation method we obtain using the Householder's iterative method of order $p$. This method is a numerical algorithm for solving the nonlinear equation $f(x)=0$, where $f(x)$ is a $p+1$ times continuously differentiable function and $\alpha$ is a zero of $f$ but not of its

2010 Mathematics Subject Classification. 11A55.

Key words and phrases. Continued fractions, Householder's iterative methods. 
derivative. Householder's method of order $p$ consists of a sequence of iterations

$$
x_{i+1}=x_{i}+p \cdot \frac{(1 / f)^{(p-1)}\left(x_{i}\right)}{(1 / f)^{(p)}\left(x_{i}\right)}
$$

beginning with an initial guess $x_{0}$. Householder's method of order 1 is just Newton's method and for Householder's method of order 2 one gets Halley's method.

In this paper we study connections between continued fraction convergents of quadratic irrational $\alpha=c+\sqrt{d}$ and Householder's iterative method of order $m-1, m \in \mathbb{N}, m \geq 2$ (with rate of convergence $m$ ) for the equation $f(x)=(x-\alpha)\left(x-\alpha^{\prime}\right)=0$, where $\alpha^{\prime}=c-\sqrt{d}$. Precisely, if the initial iteration $x_{0}=\frac{p_{n}}{q_{n}}$ is the $n$th continued fraction convergent of $\alpha$, the principal question is whether the first iteration $R_{n}^{(m)}=x_{1}$ also a convergent of $\alpha$. In that case we say that $R_{n}^{(m)}$ is a good approximant.

We will show that for quadratic irrational $\alpha$ whose period of the length $\ell$ begins with $a_{1}$, there is a good approximant at the end of the period, i.e., it holds

$$
R_{k \ell-1}^{(m)}=\frac{p_{m k \ell-1}}{q_{m k \ell-1}}, \text { for all } k \in \mathbb{N},
$$

and when period is palindromic and has even length, say $\ell=2 t$, there is a good approximant in the half of the period, i.e., it holds

$$
R_{k t-1}^{(m)}=\frac{p_{m k t-1}}{q_{m k t-1}}, \text { for all } k \in \mathbb{N} .
$$

In Section 3 we show

THEOREM 1.1. To be a good approximant is a periodic property, i.e., for all $k \in \mathbb{N}$ it holds

$$
R_{n}^{(m)}=\frac{p_{s}}{q_{s}} \quad \Longleftrightarrow \quad R_{k \ell+n}^{(m)}=\frac{p_{k m \ell+s}}{q_{k m \ell+s}},
$$

and when period is palindromic, it is also a palindromic property, i.e., it holds

$$
R_{n}^{(m)}=\frac{p_{s}}{q_{s}} \quad \Longleftrightarrow \quad R_{\ell-n-2}^{(m)}=\frac{p_{m \ell-s-2}}{q_{m \ell-s-2}} .
$$

When $\ell \leq 2$, from (1.1) and (1.2) it follows that then every approximant is good, and then it holds $R_{n}^{(m)}=\frac{p_{m(n+1)-1}}{q_{m(n+1)-1}}$ for all $n \geq 0$. So if $R_{n}^{(m)}$ is a good approximant, one might expect that it hits convergent with $m$-times larger index. However, this is not always true. If $R_{n}^{(m)}=\frac{p_{s}}{q_{s}}$, we can define numbers $j_{n}^{(m)}=j_{n}^{(m)}(\alpha)$ as half of the distance from convergent with $m$ times larger index

$$
j_{n}^{(m)}=\frac{s+1-m(n+1)}{2} .
$$


We prove that it is unbounded by constructing an explicit family of quadratic irrationals, which involves the Fibonacci numbers.

Theorem 1.2. Let $F_{\ell}$ denote the $\ell$-th Fibonacci number. Let $\ell>3, \ell \equiv$ $\pm 1(\bmod 6)$. Then for $d_{\ell}=\left(\frac{F_{\ell-3} F_{\ell}+1}{2}\right)^{2}+F_{\ell-3} F_{\ell-1}+1$ and $M \in \mathbb{N}$ it holds $\ell\left(\sqrt{d_{\ell}}\right)=\ell$ and

$$
j_{0}^{(3 M-1)}\left(\sqrt{d_{\ell}}\right)=j_{0}^{(3 M)}\left(\sqrt{d_{\ell}}\right)=j_{0}^{(3 M+1)}\left(\sqrt{d_{\ell}}\right)=\frac{\ell-3}{2} \cdot M .
$$

Connection between Newton's iterative method

$$
x_{i+1}=x_{i}-\frac{f\left(x_{i}\right)}{f^{\prime}\left(x_{i}\right)}
$$

for solving nonlinear equations $f(x)=0$ and continued fractions was discussed by several authors. So, let us briefly mention what is known in the case $m=2$.

It is well known that for $\alpha=\sqrt{d}, d \in \mathbb{N}, d$ not a perfect square, and the corresponding Newton's approximant $R_{n}^{(2)}=\frac{1}{2}\left(\frac{p_{n}}{q_{n}}+\frac{d q_{n}}{p_{n}}\right)$ it follows that (see e.g. $[1$, p. 468])

$$
R_{k \ell-1}^{(2)}=\frac{p_{2 k \ell-1}}{q_{2 k \ell-1}}, \quad \text { for } k \geq 1 .
$$

It was proved by Mikusiński [9] (see also Elezović [4]) that if $\ell=2 t$, then

$$
R_{k t-1}^{(2)}=\frac{p_{2 k t-1}}{q_{2 k t-1}}, \quad \text { for } k \geq 1 .
$$

These results imply that if $\ell(\sqrt{d}) \leq 2$, then all approximants $R_{n}^{(2)}$ are convergents of $\sqrt{d}$. In 2001, Dujella [2] proved the converse of this result. Namely, if all approximants $R_{n}^{(2)}$ are convergents of $\sqrt{d}$, then $\ell(\sqrt{d}) \leq 2$. Thus, if $\ell(\sqrt{d})>2$, we know that some of approximants $R_{n}^{(2)}$ are convergents and some of them are not. Using a result of Komatsu [8] from 1999, Dujella also showed that being a good approximant is a periodic and a palindromic property, so he defined the number $b(\sqrt{d})$ as the number of good approximants in the period. Formulas (1.4) and (1.5) suggest that $R_{n}^{(2)}$ should be convergent whose index is twice as large when it is a good approximant. However, this is not always true, and Dujella defined the number $j(\sqrt{d})$ as half of the distance from two times larger index. He also pointed out that $j(\sqrt{d})$ is unbounded. In 2005, Dujella and the author [3] proved that $b(\sqrt{d})$ is unbounded, too.

In 2011, the author [13] proved the analogous results for $\alpha=\frac{1+\sqrt{d}}{2}, d \in \mathbb{N}$, $d$ not a perfect square and $d \equiv 1(\bmod 4)$.

Sharma [15] observed arbitrary quadratic surd $\alpha=c+\sqrt{d}, c, d \in \mathbb{Q}$, $d>0, d$ is not a square of a rational number, whose continued fraction period of the length $\ell$ begins with $a_{1}$. He showed that for every such $\alpha$ and the 
corresponding Newton's approximant $R_{n}^{(2)}=\frac{p_{n}^{2}-\alpha \alpha^{\prime} q_{n}^{2}}{q_{n}\left(2 p_{n}-\left(\alpha+\alpha^{\prime}\right) q_{n}\right)}$ it holds

$$
R_{k \ell-1}^{(2)}=\frac{p_{2 k \ell-1}}{q_{2 k \ell-1}}, \quad \text { for } k \geq 1,
$$

and when $\ell=2 t$ and the period is palindromic then it holds

$$
R_{k t-1}^{(2)}=\frac{p_{2 k t-1}}{q_{2 k t-1}}, \quad \text { for } k \geq 1 .
$$

Frank and Sharma [6] discussed generalization of Newton's formula. They showed that for every quadratic irrational $\alpha$, whose period begins with $a_{1}$, it holds

(1.6) $\frac{p_{m k \ell-1}}{q_{m k \ell-1}}=\frac{\alpha\left(p_{k \ell-1}-\alpha^{\prime} q_{k \ell-1}\right)^{m}-\alpha^{\prime}\left(p_{k \ell-1}-\alpha q_{k \ell-1}\right)^{m}}{\left(p_{k \ell-1}-\alpha^{\prime} q_{k \ell-1}\right)^{m}-\left(p_{k \ell-1}-\alpha q_{k \ell-1}\right)^{m}}$, for $k, m \in \mathbb{N}$, and when $\ell=2 t$ and the period is palindromic then it holds

$$
\frac{p_{m k t-1}}{q_{m k t-1}}=\frac{\alpha\left(p_{k t-1}-\alpha^{\prime} q_{k t-1}\right)^{m}-\alpha^{\prime}\left(p_{k t-1}-\alpha q_{k t-1}\right)^{m}}{\left(p_{k t-1}-\alpha^{\prime} q_{k t-1}\right)^{m}-\left(p_{k t-1}-\alpha q_{k t-1}\right)^{m}}, \text { for } k, m \in \mathbb{N} \text {. }
$$

\section{Householder's Methods}

Householder's iterative method (see [14], [7, §4.4]) of order $p$ for rootsolving, consists of a sequence of iterations

$$
x_{i+1}=H^{(p)}\left(x_{i}\right)=x_{i}+p \cdot \frac{(1 / f)^{(p-1)}\left(x_{i}\right)}{(1 / f)^{(p)}\left(x_{i}\right)},
$$

(where $(1 / f)^{(p)}$ denotes $p$-th derivative of $1 / f$ ) beginning with an initial guess $x_{0}$. Let $f(x)$ be a $p+1$ times continuously differentiable function and $\alpha$ is a zero of $f$ but not of its derivative, then, in a neighborhood of $\alpha$, the convergence has rate $p+1$.

Analogous to Newton's method, we will start with function $f(x)=(x-$ $\alpha)\left(x-\alpha^{\prime}\right)$, which satisfies the above conditions. Let us first observe $p$-th derivative of the function $1 / f$

$$
\begin{aligned}
(1 / f)^{(p)} & =\left(\frac{1}{(x-\alpha)\left(x-\alpha^{\prime}\right)}\right)^{(p)}=\frac{1}{\alpha-\alpha^{\prime}}\left(\frac{1}{x-\alpha}-\frac{1}{x-\alpha^{\prime}}\right)^{(p)} \\
& =\frac{(-1)^{p} p !}{\alpha-\alpha^{\prime}}\left(\frac{1}{(x-\alpha)^{p+1}}-\frac{1}{\left(x-\alpha^{\prime}\right)^{p+1}}\right) .
\end{aligned}
$$

So we have

$$
\begin{aligned}
H^{(p)}(x) & =x-\frac{\left(x-\alpha^{\prime}\right)^{p}-(x-\alpha)^{p}}{\left(x-\alpha^{\prime}\right)^{p+1}-(x-\alpha)^{p+1}}(x-\alpha)\left(x-\alpha^{\prime}\right) \\
& =\frac{\alpha\left(x-\alpha^{\prime}\right)^{p+1}-\alpha^{\prime}(x-\alpha)^{p+1}}{\left(x-\alpha^{\prime}\right)^{p+1}-(x-\alpha)^{p+1}} .
\end{aligned}
$$


It is not hard to show that it holds

$$
H^{(p+1)}(x)=\frac{x H^{(p)}(x)-\alpha \alpha^{\prime}}{H^{(p)}(x)+x-\alpha-\alpha^{\prime}}, \quad \text { for } p \in \mathbb{N} .
$$

Formula (1.6) shows that for an arbitrary quadratic surd, whose period begins with $a_{1}$ and $k \in \mathbb{N}, m=2,3, \ldots$, it holds

$$
H^{(m-1)}\left(\frac{p_{k \ell-1}}{q_{k \ell-1}}\right)=\frac{p_{m k \ell-1}}{q_{m k \ell-1}}
$$

and when period is palindromic, and has even length, say $\ell=2 t$, from (1.7) it follows

$$
H^{(m-1)}\left(\frac{p_{k t-1}}{q_{k t-1}}\right)=\frac{p_{m k t-1}}{q_{m k t-1}} .
$$

Let us recall the definition

$$
R_{n}^{(1)}=\frac{p_{n}}{q_{n}}, \quad \text { and for } m>1 \quad R_{n}^{(m)}=H^{(m-1)}\left(\frac{p_{n}}{q_{n}}\right),
$$

and we say that $R_{n}^{(m)}$ is good approximation, if it is a convergent of $\alpha$. From (2.3) and (2.4) it follows (1.1) and (1.2). From (2.1) we have

$$
R_{n}^{(m)}=\frac{\alpha\left(p_{n}-\alpha^{\prime} q_{n}\right)^{m}-\alpha^{\prime}\left(p_{n}-\alpha q_{n}\right)^{m}}{\left(p_{n}-\alpha^{\prime} q_{n}\right)^{m}-\left(p_{n}-\alpha q_{n}\right)^{m}},
$$

and formula (2.2) says

$$
R_{n}^{(m+1)}=\frac{R_{n}^{(1)} R_{n}^{(m)}-\alpha \alpha^{\prime}}{R_{n}^{(1)}+R_{n}^{(m)}-\alpha-\alpha^{\prime}}, \quad \text { for } m \in \mathbb{N}, n=0,1, \ldots
$$

\section{GOOD APPROXIMANTS ARE PERIODIC AND PALINDROMIC}

From now on, we assume that $\alpha$ is quadratic irrational whose period of the length $\ell$ begins with $a_{1}$. From formula [15, (8)] we obtain

$$
\begin{aligned}
\left(a_{\ell}-a_{0}\right) p_{k \ell-1}+p_{k \ell-2} & =-\alpha \alpha^{\prime} q_{k \ell-1}, \\
\left(a_{\ell}-a_{0}\right) q_{k \ell-1}+q_{k \ell-2} & =p_{k \ell-1}-\left(\alpha+\alpha^{\prime}\right) q_{k \ell-1},
\end{aligned}
$$

for all $k \in \mathbb{N}$.

Lemma 3.1. For $m, k \in \mathbb{N}$ and $i=1,2, \ldots, \ell$, it holds

$$
R_{k \ell+i-1}^{(m)}=\frac{R_{k \ell-1}^{(m)} R_{i-1}^{(m)}-\alpha \alpha^{\prime}}{R_{k \ell-1}^{(m)}+R_{i-1}^{(m)}-\alpha-\alpha^{\prime}} .
$$

Proof. For $m=1$, statement of the lemma is proven in [5, Thm. 2.1]. Suppose that (3.3) holds for some $m \in \mathbb{N}$, and let us show that it holds for 
$m+1$ too. Using the notation $s=R_{k \ell-1}^{(1)}, S=R_{k \ell-1}^{(m)}, t=R_{i-1}^{(1)}$ and $T=R_{i-1}^{(m)}$, we have

$$
\begin{aligned}
& R_{k \ell+i-1}^{(m+1)} \stackrel{(2.6)}{=} \frac{R_{k \ell+i-1}^{(1)} R_{k \ell+i-1}^{(m)}-\alpha \alpha^{\prime}}{R_{k \ell+i-1}^{(1)}+R_{k \ell+i-1}^{(m)}-\alpha-\alpha^{\prime}}=\frac{\frac{s t-\alpha \alpha^{\prime}}{s+t-\alpha-\alpha^{\prime}} \cdot \frac{S T-\alpha \alpha^{\prime}}{S+T-\alpha-\alpha^{\prime}}-\alpha \alpha^{\prime}}{\frac{s t-\alpha \alpha^{\prime}}{s+t-\alpha-\alpha^{\prime}}+\frac{S T-\alpha \alpha^{\prime}}{S+T-\alpha-\alpha^{\prime}}-\alpha-\alpha^{\prime}} \\
& \quad= \frac{\left(s t-\alpha \alpha^{\prime}\right)\left(S T-\alpha \alpha^{\prime}\right)-\alpha \alpha^{\prime}\left(s+t-\alpha-\alpha^{\prime}\right)\left(S+T-\alpha-\alpha^{\prime}\right)}{\left(s t-\alpha \alpha^{\prime}\right)\left(S+T-\alpha-\alpha^{\prime}\right)+\left(S T-\alpha \alpha^{\prime}\right)\left(s+t-\alpha-\alpha^{\prime}\right)-\left(\alpha+\alpha^{\prime}\right)\left(s+t-\alpha-\alpha^{\prime}\right)\left(S+T-\alpha-\alpha^{\prime}\right)} \\
& \quad= \frac{\left(s S-\alpha \alpha^{\prime}\right)\left(t T-\alpha \alpha^{\prime}\right)-\alpha \alpha^{\prime}\left(s+S-\alpha-\alpha^{\prime}\right)\left(t+T-\alpha-\alpha^{\prime}\right)}{\left(s S-\alpha \alpha^{\prime}\right)\left(t+T-\alpha-\alpha^{\prime}\right)+\left(t T-\alpha \alpha^{\prime}\right)\left(s+S-\alpha-\alpha^{\prime}\right)-\left(\alpha+\alpha^{\prime}\right)\left(s+S-\alpha-\alpha^{\prime}\right)\left(t+T-\alpha-\alpha^{\prime}\right)} \\
& \quad=\frac{\frac{s S-\alpha \alpha^{\prime}}{s+S-\alpha-\alpha^{\prime}} \cdot \frac{t T-\alpha \alpha^{\prime}}{t+T-\alpha-\alpha^{\prime}}-\alpha \alpha^{\prime}}{\frac{s S-\alpha \alpha^{\prime}}{s+S-\alpha-\alpha^{\prime}}+\frac{t T-\alpha \alpha^{\prime}}{t+T-\alpha-\alpha^{\prime}}-\alpha-\alpha^{\prime}} \stackrel{(2.6)}{=} \frac{R_{k \ell-1}^{(m+1)} R_{i-1}^{(m+1)}-\alpha \alpha^{\prime}}{R_{k \ell-1}^{(m+1)}+R_{i-1}^{(m+1)}-\alpha-\alpha^{\prime}} .
\end{aligned}
$$

We have (see e.g. $[12, \S 23])-\left(\alpha-a_{0}\right)^{\prime}=\left[\overline{a_{\ell}, a_{\ell-1}, \ldots, a_{2}, a_{1}}\right]$, and so

$$
\frac{1}{a_{0}-a_{\ell}-\alpha^{\prime}}=\left[\overline{a_{\ell-1}, \ldots, a_{2}, a_{1}, a_{\ell}}\right]
$$

So when period is palindromic, we have $\alpha+\alpha^{\prime}=2 a_{0}-a_{\ell}$, thus formulas (3.1) and (3.2) in palindromic case become

$$
\begin{aligned}
a_{0} p_{k \ell-1}+p_{k \ell-2} & =\left(\alpha+\alpha^{\prime}\right) p_{k \ell-1}-\alpha \alpha^{\prime} q_{k \ell-1}, \\
a_{0} q_{k \ell-1}+q_{k \ell-2} & =p_{k \ell-1} .
\end{aligned}
$$

Lemma 3.2. For $m, k \in \mathbb{N}$ and $i=1,2, \ldots, \ell-1$, when period is palindromic, it holds

$$
R_{k \ell-i-1}^{(m)}=\frac{R_{k \ell-1}^{(m)}\left(R_{i-1}^{(m)}-\alpha-\alpha^{\prime}\right)+\alpha \alpha^{\prime}}{R_{i-1}^{(m)}-R_{k \ell-1}^{(m)}} .
$$

Proof. For $m=1$ we have

$$
\begin{aligned}
& R_{k \ell-i-1}^{(1)}=\frac{p_{k \ell-i-1}}{q_{k \ell-i-1}}=\frac{0 \cdot p_{k \ell-i}+p_{k \ell-i-1}}{0 \cdot q_{k \ell-i}+q_{k \ell-i-1}}=\left[a_{0}, a_{1}, \ldots, a_{k \ell-i-1}, a_{k \ell-i}, 0\right] \\
& =\left[a_{0}, a_{1}, \ldots, a_{k \ell-i}, a_{k \ell-i+1}, \ldots, a_{k \ell-1}, a_{0}, 0,-a_{0},-a_{1}, \ldots,-a_{i-1}\right] \\
& =\left[a_{0}, a_{1}, \ldots, a_{k \ell-i}, a_{k \ell-i+1}, \ldots, a_{k \ell-1}, a_{0}-\frac{p_{i-1}}{q_{i-1}}\right] \\
& =\frac{p_{k \ell-1}\left(a_{0}-R_{i-1}^{(1)}\right)+p_{k \ell-2}}{q_{k \ell-1}\left(a_{0}-R_{i-1}^{(1)}\right)+q_{k \ell-2}} \underset{(3.5)}{\stackrel{(3.4)}{=}} \frac{R_{k \ell-1}^{(1)}\left(R_{i-1}^{(1)}-\alpha-\alpha^{\prime}\right)+\alpha \alpha^{\prime}}{R_{i-1}^{(1)}-R_{k \ell-1}^{(1)}} .
\end{aligned}
$$


Suppose that (3.6) holds for some $m \in \mathbb{N}$, and let us show that it holds for $m+1$ too. With the same notation as in the proof of Lemma 3.1, we have

$$
\begin{aligned}
R_{k \ell-i-1}^{(m+1)} & \stackrel{(2.6)}{=} \frac{R_{k \ell-i-1}^{(1)} R_{k \ell-i-1}^{(m)}-\alpha \alpha^{\prime}}{R_{k \ell-i-1}^{(1)}+R_{k \ell-i-1}^{(m)}-\alpha-\alpha^{\prime}} \\
& =\frac{\frac{s\left(t-\alpha-\alpha^{\prime}\right)+\alpha \alpha^{\prime}}{t-s} \cdot \frac{S\left(T-\alpha-\alpha^{\prime}\right)+\alpha \alpha^{\prime}}{T-S}-\alpha \alpha^{\prime}}{\frac{s\left(t-\alpha-\alpha^{\prime}\right)+\alpha \alpha^{\prime}}{t-s}+\frac{S\left(T-\alpha-\alpha^{\prime}\right)+\alpha \alpha^{\prime}}{T-S}-\alpha-\alpha^{\prime}} \\
& =\frac{\left(s\left(t-\alpha-\alpha^{\prime}\right)+\alpha \alpha^{\prime}\right)\left(S\left(T-\alpha-\alpha^{\prime}\right)+\alpha \alpha^{\prime}\right)-\alpha \alpha^{\prime}(t-s)(T-S)}{\left(s\left(t-\alpha-\alpha^{\prime}\right)+\alpha \alpha^{\prime}\right)(T-S)+\left(S\left(T-\alpha-\alpha^{\prime}\right)+\alpha \alpha^{\prime}\right)(t-s)-\left(\alpha+\alpha^{\prime}\right)(t-s)(T-S)} \\
& =\frac{\left(s S-\alpha \alpha^{\prime}\right)\left(t T-\alpha \alpha^{\prime}-\left(\alpha+\alpha^{\prime}\right)\left(t+T-\alpha-\alpha^{\prime}\right)\right)+\alpha \alpha^{\prime}\left(s+S-\alpha-\alpha^{\prime}\right)\left(t+T-\alpha-\alpha^{\prime}\right)}{\left(t T-\alpha \alpha^{\prime}\right)\left(s+S-\alpha-\alpha^{\prime}\right)-\left(s S-\alpha \alpha^{\prime}\right)\left(t+T-\alpha-\alpha^{\prime}\right)} \\
& =\frac{\frac{s S-\alpha \alpha^{\prime}}{s+S-\alpha-\alpha^{\prime}}\left(\frac{t T-\alpha \alpha^{\prime}}{t+T-\alpha-\alpha^{\prime}}-\alpha-\alpha^{\prime}\right)+\alpha \alpha^{\prime}}{\frac{t T-\alpha \alpha^{\prime}}{t+T-\alpha-\alpha^{\prime}}-\frac{s S-\alpha \alpha^{\prime}}{s+S-\alpha-\alpha^{\prime}}} \\
& \stackrel{(2.6)}{=} \frac{R_{k \ell-1}^{(m+1)}\left(R_{i-1}^{(m+1)}-\alpha-\alpha^{\prime}\right)+\alpha \alpha^{\prime}}{R_{i-1}^{(m+1)}-R_{k \ell-1}^{(m+1)}} .
\end{aligned}
$$

Let us show that each approximant can be expressed as the combination of convergent with $m$ times larger index and carefully selected numbers $\beta_{i}^{(m)}$, which are periodic (so we take them for $i=1, \ldots \ell-1$ ).

Proposition 3.3. Let $m \in \mathbb{N}$. For $i=1,2, \ldots, \ell-1$ let

Then it holds

$$
\beta_{i}^{(m)}=-\frac{p_{m i-1}-R_{i-1}^{(m)} q_{m i-1}}{p_{m i}-R_{i-1}^{(m)} q_{m i}} .
$$

$$
R_{k \ell+i-1}^{(m)}=\frac{\beta_{i}^{(m)} p_{m(k \ell+i)}+p_{m(k \ell+i)-1}}{\beta_{i}^{(m)} q_{m(k \ell+i)}+q_{m(k \ell+i)-1}}, \text { for all } k \geq 0,
$$

and when period is palindromic, then

$$
R_{k \ell-i-1}^{(m)}=\frac{p_{m(k \ell-i)-1}-\beta_{i}^{(m)} p_{m(k \ell-i)-2}}{q_{m(k \ell-i)-1}-\beta_{i}^{(m)} q_{m(k \ell-i)-2}} \text {, for all } k \geq 1 \text {. }
$$

Proof. Let us first consider the continued fraction expansion of $\beta_{i}^{(m)}$.

$$
\begin{aligned}
\beta_{i}^{(m)} & =-\left[0, \frac{p_{m i}-R_{i-1}^{(m)} q_{m i}}{p_{m i-1}-R_{i-1}^{(m)} q_{m i-1}}\right] \\
\stackrel{[13,}{ } & ={ }^{\text {Lm. 3] }}-\left[0, a_{m i}, a_{m i-1}, \ldots, a_{1}, a_{0}-R_{i-1}^{(m)}\right] \\
& =\left[0,-a_{m i},-a_{m i-1}, \ldots,-a_{1},-a_{0}+R_{i-1}^{(m)}\right] .
\end{aligned}
$$


If $k=0$ we have

$$
\begin{aligned}
& \frac{\beta_{i}^{(m)} p_{m i}+p_{m i-1}}{\beta_{i}^{(m)} q_{m i}+q_{m i-1}}=\left[a_{0}, a_{1}, \ldots, a_{m i-1}, a_{m i}, \beta_{i}^{(m)}\right] \\
& \quad=\left[a_{0}, a_{1}, \ldots, a_{m i-1}, a_{m i}, 0,-a_{m i},-a_{m i-1}, \ldots,-a_{1},-a_{0}+R_{i-1}^{(m)}\right]=R_{i-1}^{(m)},
\end{aligned}
$$

and if $k>0$ we have

$$
\begin{aligned}
& \frac{\beta_{i}^{(m)} p_{m(k \ell+i)}+p_{m(k \ell+i)-1}}{\beta_{i}^{(m)} q_{m(k \ell+i)}+q_{m(k \ell+i)-1}} \\
& =\left[a_{0}, a_{1}, \ldots, a_{m k \ell-1}, a_{m k \ell}, a_{m k \ell+1}, \ldots, a_{m(k \ell+i)}, \beta_{i}^{(m)}\right] \\
& =\left[a_{0}, a_{1}, \ldots, a_{m k \ell-1}, a_{m k \ell}-a_{0}+R_{i-1}^{(m)}\right] \\
& =\frac{p_{m k \ell-1}\left(a_{m k \ell}-a_{0}+R_{i-1}^{(m)}\right)+p_{m k \ell-2}}{q_{m k \ell-1}\left(a_{m k \ell}-a_{0}+R_{i-1}^{(m)}\right)+q_{m k \ell-2}} \\
& \stackrel{(3.1)}{=} \frac{p_{m k \ell-1} R_{i-1}^{(m)}-\alpha \alpha^{\prime} q_{m k \ell-1}}{p_{m k \ell-1}+q_{m k \ell-1}\left(R_{i-1}^{(m)}-\alpha-\alpha^{\prime}\right)} \\
& \stackrel{(2.3)}{=} \frac{R_{k \ell-1}^{(m)} R_{i-1}^{(m)}-\alpha \alpha^{\prime}}{R_{k \ell-1}^{(m)}+R_{i-1}^{(m)}-\alpha-\alpha^{\prime}} \stackrel{\text { Lm. }}{=}{ }^{.1} R_{k \ell+i-1}^{(m)} \text {. }
\end{aligned}
$$

When period is palindromic we have

$$
\begin{aligned}
& \frac{p_{m(k \ell-i)-1}-\beta_{i}^{(m)} p_{m(k \ell-i)-2}}{q_{m(k \ell-i)-1}-\beta_{i}^{(m)} q_{m(k \ell-i)-2}}=\left[a_{0}, a_{1}, \ldots, a_{m(k \ell-i)-1},-\frac{1}{\beta_{i}^{(m)}}\right] \\
& =\left[a_{0}, a_{1}, \ldots, a_{m(k \ell-i)-1}, 0,0, a_{m i}, a_{m i-1}, \ldots, a_{1}, a_{0}-R_{i-1}^{(m)}\right] \\
& =\left[a_{0}, a_{1}, \ldots, a_{m(k \ell-i)-1}, a_{m(k \ell-i)}, a_{m(k \ell-i)+1}, \ldots, a_{m k \ell-1}, a_{0}-R_{i-1}^{(m)}\right] \\
& =\frac{p_{m k \ell-1}\left(a_{0}-R_{i-1}^{(m)}\right)+p_{m k \ell-2}}{q_{m k \ell-1}\left(a_{0}-R_{i-1}^{(m)}\right)+q_{m k \ell-2}} \stackrel{(3.4)}{=} \frac{p_{m k \ell-1}\left(R_{i-1}^{(m)}-\alpha-\alpha^{\prime}\right)+\alpha \alpha^{\prime} q_{m k \ell-1}}{q_{m k \ell-1} R_{i-1}^{(m)}-p_{m k \ell-1}} \\
& \stackrel{(2.3)}{=} \frac{R_{k \ell-1}^{(m)}\left(R_{i-1}^{(m)}-\alpha-\alpha^{\prime}\right)+\alpha \alpha^{\prime}}{R_{i-1}^{(m)}-R_{k \ell-1}^{(m)}} \stackrel{\operatorname{Lm.3.2}^{\prime}}{=} R_{k \ell-i-1}^{(m)} .
\end{aligned}
$$

REMARK 3.4. [8, Thm.1] and [13, Thm.2] are special cases of the last proposition for $m=2$ and $\alpha=\sqrt{d}$ and $\alpha=\frac{1+\sqrt{d}}{2}$, respectively.

Proof of Thereom 1.1. The first part for $n=\ell-1$ is (1.1) and for $n=0,1, \ldots, \ell-2$ follows from (3.7). The second part follows similarly as in $\left[2\right.$, Lm. 3], but we have three cases. Let $R_{n}^{(m)}=\frac{p_{s}}{q_{s}}=\left[a_{0}, a_{1}, \ldots, a_{s}\right]$. 
If $s=m(n+1)-1$, from (3.7) we have $\beta_{n+1}^{(m)}=0$, so from (3.8) we have $R_{\ell-n-2}^{(m)}=\frac{p_{m(\ell-n-1)-1}}{q_{m(\ell-n-1)-1}}=\frac{p_{m \ell-s-2}}{q_{m \ell-s-2}}$.

If $s>m(n+1)-1$, then from (3.7) we have

$$
\begin{aligned}
\beta_{n+1}^{(m)} & =\left[a_{m(n+1)+1}, a_{m(n+1)+2}, \ldots, a_{s}\right] \\
& =\left[a_{m(\ell-n-1)-1}, a_{m(\ell-n-1)-2}, \ldots, a_{m \ell-s}\right] .
\end{aligned}
$$

From (3.8) we have

$$
\begin{aligned}
& R_{\ell-n-2}^{(m)}=\frac{p_{m(\ell-n-1)-1}-\beta_{n+1}^{(m)} p_{m(\ell-n-1)-2}}{q_{m(\ell-n-1)-1}-\beta_{n+1}^{(m)} q_{m(\ell-n-1)-2}} \\
& =\left[a_{0}, a_{1}, \ldots, a_{m(\ell-n-1)-1},-\frac{1}{\beta_{n+1}^{(m)}}\right] \\
& =\left[a_{0}, a_{1}, \ldots, a_{m(\ell-n-1)-1}, 0,-a_{m(\ell-n-1)-1},-a_{m(\ell-n-1)-2}, \ldots,-a_{m \ell-s}\right] \\
& =\left[a_{0}, a_{1}, \ldots, a_{m \ell-s-1}, 0\right]=\frac{p_{m \ell-s-2}}{q_{m \ell-s-2}} . \\
& \quad \text { If } s<m(n+1)-1, \text { then from }(3.8) \text { we have } \\
& \beta_{\ell-n-1}^{(m)}=\left[a_{m(n+1)-1}, a_{m(n+1)-2}, \ldots, a_{s+2}\right] \\
& =\left[a_{m(\ell-n-1)+1}, a_{m(\ell-n-1)+2}, \ldots, a_{m \ell-s-2}\right] .
\end{aligned}
$$

From (3.7) we have

$$
R_{\ell-n-2}^{(m)}=\frac{\beta_{\ell-n-1}^{(m)} p_{m(\ell-n-1)}+p_{m(\ell-n-1)-1}}{\beta_{\ell-n-1}^{(m)} q_{m(\ell-n-1)}+q_{m(\ell-n-1)-1}}=\frac{p_{m \ell-s-2}}{q_{m \ell-s-2}} .
$$

Let us show how Theorem 1.1 can be applied. The first example shows palindromic situation, the second is not palindromic (but we accidentally get good approximation in the half of the period), and the third shows that good approximants do depend on $m$.

EXAMPLE 3.5. Let us observe $\sqrt{44}=[6, \overline{1,1,1,2,1,1,1,12}]$. The period is palindromic and we have $\ell=8$. Let us consider e.g. the case $m=5$. From (2.5) we have

$$
R_{n}^{(5)}=\frac{p_{n}^{5}+440 p_{n}^{3} q_{n}^{2}+9680 p_{n} q_{n}^{4}}{5 p_{n}^{4} q_{n}+440 p_{n}^{2} q_{n}^{3}+1936 q_{n}^{5}} .
$$

From (1.2) we have

$$
\begin{aligned}
& R_{3}^{(5)}=\frac{p_{19}}{q_{19}}=\frac{3160100}{476403}, R_{7}^{(5)}=\frac{p_{39}}{q_{39}}=\frac{4993116004999}{752740560150}, \\
& R_{11}^{(5)}=\frac{p_{59}}{q_{59}}, R_{15}^{(5)}=\frac{p_{79}}{q_{79}}, \ldots, R_{4 k-1}^{(5)}=\frac{p_{20 k-1}}{q_{20 k-1}} .
\end{aligned}
$$


Since, $R_{0}^{(5)}=\frac{p_{8}}{q_{8}}=\frac{2514}{379}$. From Theorem 1.1 we have $R_{6}^{(5)}=\frac{p_{30}}{q_{30}}=\frac{7944493914}{1197677521}$, and also $R_{8 k}^{(5)}=\frac{p_{40 k+8}}{q_{40 k+8}}$ and $R_{8 k-2}^{(5)}=\frac{p_{40 k-10}}{q_{40 k-10}}$. be.

$R_{1}^{(5)}=\frac{235487}{35501}$ is not a convergent of $\sqrt{44}$, so neither $R_{8 k+1}^{(5)}$ nor $R_{8 k-3}^{(5)}$ will will be.

$R_{2}^{(5)}=\frac{6251453}{942442}$ is not a convergent of $\sqrt{44}$, so neither $R_{8 k+2}^{(5)}$ nor $R_{8 k-4}^{(5)}$

EXAMPLE 3.6. Let us observe $\alpha=\frac{5+\sqrt{21}}{3}=[9, \overline{5,6,1,2}]$ and $m=3$. From 2.5 we have $R_{m}^{(3)}=\frac{37 p_{n}^{3}-4572 p_{n} q_{n}^{2}+23368 q_{n}^{3}}{81 p_{n}^{2} q_{n}-1242 p_{n} q_{n}^{2}+4824 q_{n}^{3}}$.

We have $R_{3}^{(3)}=\frac{p_{11}}{q_{11}}=\frac{44004659}{435564}$, and so $R_{4 k-1}^{(3)}=\frac{p_{12 k-1}}{q_{12 k-1}}$. The period is not palindromic, and accidentally we have: $R_{1}^{(3)}=\frac{p_{7}}{q_{7}}=\frac{36409}{3960}$ (in palindromic case it would be $\frac{p_{5}}{q_{5}}$ ), and so $R_{4 k+1}^{(3)}=\frac{p_{12 k+7}}{q_{12 k+7}}$.

EXAMPLE 3.7. Let us observe $\alpha=\frac{7+\sqrt{11}}{5}=[2, \overline{15,1,3,1,3,1}]$. For $m=3$ we have $R_{6 k-1}^{(3)}=\frac{p_{18 k-1}}{q_{18 k-1}} ; R_{1}^{(3)}=\frac{p_{7}}{q_{7}}$ and $R_{6 k+1}^{(3)}=\frac{p_{18 k+7}}{q_{18 k+7}}$.

For $m=4$ we have: $R_{6 k-1}^{(4)}=\frac{p_{24 k-1}}{q_{24 k-1}} ; R_{0}^{(4)}=\frac{p_{5}}{q_{5}}$ and $R_{6 k}^{(4)}=\frac{p_{24 k+5}}{q_{24 k+5}}$; $R_{1}^{(4)}=\frac{p_{11}}{q_{11}}$ and $R_{6 k+1}^{(4)}=\frac{p_{24 k+11}}{q_{24 k+11}} ; R_{3}^{(4)}=\frac{p_{17}}{q_{17}}$ and $R_{6 k+3}^{(4)}=\frac{p_{24 k+17}}{q_{24 k+17}}$.

\section{WHICH CONVERGENTS MAY APPEAR?}

From now on, let us observe only quadratic irrationals of the form $\alpha=\sqrt{d}$, $d \in \mathbb{N}, d$ not a perfect square. It is well known that period of such $\alpha$ begins with $a_{1}$ and is palindromic.

LEMMA 4.1. $\quad$ a) $R_{n}^{(m)}<\sqrt{d}$ if and only if $n$ is even and $m$ is odd. Therefore, $R_{n}^{(m)}$ can be an even convergent only if $n$ is even and $m$ is odd.

b)

$$
\left|R_{n+1}^{(m)}-\sqrt{d}\right|<\left|R_{n}^{(m)}-\sqrt{d}\right| .
$$

Proof. a) From (2.5) we have

$$
R_{n}^{(m)}-\sqrt{d}=\frac{2 \sqrt{d}\left(p_{n}-q_{n} \sqrt{d}\right)^{m}}{\left(p_{n}+q_{n} \sqrt{d}\right)^{m}-\left(p_{n}-q_{n} \sqrt{d}\right)^{m}} .
$$

On the right side of (4.2), denominator is always greater than 0 , and nominator is less than 0 if and only if $n$ is even (then we have $p_{n}-\sqrt{d} q_{n}<0$ ) and $m$ is odd.

b) Let us observe (4.2). From $1 \leq p_{0}<p_{1}<p_{2}<\ldots$ and $1 \leq q_{0}<q_{1}<$ $q_{2}<\ldots$ we have $2<p_{0}+\sqrt{d} q_{0}<p_{1}+\sqrt{d} q_{1}<\ldots$ On the other hand, we have $1>\left|p_{0}-\sqrt{d} q_{0}\right|>\left|p_{1}-\sqrt{d} q_{1}\right|>\ldots$ (see e.g. $[12, \S 15]$ ), so it holds (4.1). 
Let us observe the definition (1.3). The number $j_{n}^{(m)}$ is an integer, by Lemma 4.1 a). Using Theorem 1.1 we have

$$
j_{n}^{(m)}=j_{k \ell+n}^{(m)} \text {, and in palindromic case } j_{n}^{(m)}=-j_{\ell-n-2}^{(m)} \text {. }
$$

Proposition 4.2. For $n \geq 0$ and $m \in \mathbb{N}$ we have

$$
\left|j_{n}^{(m)}(\sqrt{d})\right|<\frac{m(\ell / 2-1)}{2} .
$$

Proof. Let $R_{n}^{(m)}=\frac{p_{m(n+1)+2 j-1}}{q_{m(n+1)+2 j-1}}$. According to (4.3), it suffices to consider the case $j>0$ and $n<\ell$.

Assume first that $\ell$ is even, that is $\ell=2 t$. We have $R_{t-1}^{(m)}=\frac{p_{m t-1}}{q_{m t-1}}$ and $R_{\ell-1}^{(m)}=\frac{p_{m \ell-1}}{q_{m \ell-1}}$. For $n<t-1$, using (4.1) we have $m(n+1)+2 j-1<m t-1$, and $2 j \leq m(t-1)-1$. For $n=t-1$ and $n=\ell-1$ we have $j=0$, and for $t-1<n<\ell-1$ we have $m(n+1)+2 j-1<m \ell-1$, or $2 j<m \ell-m(n+1) \leq$ $m(t-1)$, so again we get $j \leq \frac{m(\ell / 2-1)-1}{2}$.

Let $\ell$ is odd, e.g. $\ell=2 t+1$. If for some $n, 0 \leq n<t$ holds $j \geq \frac{m(\ell / 2-1)}{2}$, we would have $s:=m(n+1)+2 j-1 \geq m \ell / 2-1$. By Theorem 1.1 it follows $R_{\ell-n-2}^{(m)}=\frac{p_{m \ell-s-2}}{q_{m \ell-s-2}}$, and $m \ell-s-2 \leq m \ell / 2-1$. Now it holds $\left|\sqrt{d}-\frac{p_{s}}{q_{s}}\right| \leq\left|\sqrt{d}-\frac{p_{m \ell-s-2}}{q_{m \ell-s-2}}\right|$, thus $\left|\sqrt{d}-R_{n}^{(m)}\right| \leq\left|\sqrt{d}-R_{\ell-n-2}^{(m)}\right|$. This is not possible by (4.1), since $\ell-n-2 \geq t$. For $t-1<n<\ell-1$, the proof is the same as in the even case.

Proposition 4.3. Let $\ell \in \mathbb{N}$ and $a_{1}, \ldots, a_{\ell-1} \in \mathbb{N}$ such that $a_{1}=a_{\ell-1}$, $a_{2}=a_{\ell-2}, \ldots$ The number $\left[a_{0}, \overline{a_{1}, a_{2}, \ldots, a_{\ell-1}, 2 a_{0}}\right]$ is of the form $\sqrt{d}$, $d \in \mathbb{N}$ if and only if

$$
2 a_{0} \equiv(-1)^{\ell-1} p_{\ell-2}^{\prime} q_{\ell-2}^{\prime} \quad\left(\bmod p_{\ell-1}^{\prime}\right),
$$

where $\frac{p_{n}^{\prime}}{q_{n}^{\prime}}=\left[a_{1}, a_{2}, \ldots, a_{n-1}, a_{n}\right]$. Then it holds

$$
d=a_{0}^{2}+\frac{2 a_{0} p_{\ell-2}^{\prime}+q_{\ell-2}^{\prime}}{p_{\ell-1}^{\prime}} .
$$

Proof. See $[12, \S 26]$

Lemma 4.4. Let $F_{k}$ denote the $k$-th Fibonacci number. Let $n \in \mathbb{N}$ and $k>1, k \equiv 1,2(\bmod 3)$. For $d_{k}(n)=\left(\frac{(2 n-1) F_{k}+1}{2}\right)^{2}+(2 n-1) F_{k-1}+1$ it holds $\ell\left(\sqrt{d_{k}(n)}\right)=k$ and

$$
\sqrt{d_{k}(n)}=[\frac{(2 n-1) F_{k}+1}{2}, \underbrace{\overline{1,1, \ldots, 1,1},(2 n-1) F_{k}+1}_{k-1 \text { times }}] .
$$

Proof. From (4.4), it follows

$$
2 a_{0} \equiv(-1)^{k-1} F_{k-1} F_{k-2} \equiv(-1)^{k-1} F_{k-1}\left(F_{k}-F_{k-1}\right)
$$




$$
\equiv(-1)^{k-1}\left(-F_{k-1}^{2}\right) \quad\left(\bmod F_{k}\right) .
$$

Now from Cassini's identity $F_{k} F_{k-2}-F_{k-1}^{2}=(-1)^{k-1}$ we have $2 a_{0} \equiv 1$ $\left(\bmod F_{k}\right)$. When $3 \mid k$, this congruence is not solvable, and if $3 \nmid k$, the solution is $a_{0} \equiv \frac{F_{k}+1}{2}\left(\bmod F_{k}\right)$, i.e.,

$$
a_{0}=\frac{F_{k}+1}{2}+(n-1) F_{k}=\frac{(2 n-1) F_{k}+1}{2}, \quad n \in \mathbb{N} .
$$

From (4.5) it follows

$$
\begin{aligned}
d & =\left(\frac{(2 n-1) F_{k}+1}{2}\right)^{2}+\frac{\left((2 n-1) F_{k}+1\right) F_{k-1}+F_{k-2}}{F_{k}} \\
& =\left(\frac{(2 n-1) F_{k}+1}{2}\right)^{2}+(2 n-1) F_{k-1}+1 .
\end{aligned}
$$

Remark 4.5. Periodic continued fractions involving Fibonacci numbers with all $a_{i}=1, i=1, \ldots, \ell-1$ were known earlier. First example was shown in [16]. Construction of such examples, using Pellian equations was given in several papers by Mollin, see e.g. [11] and [10]. However, in all such examples, the numbers are of the form $\frac{1+\sqrt{d}}{2}$ with all $a_{i}=1$ in symmetric part of the period, but for the numbers of the form $\sqrt{d}$ there is at least one $a_{i} \neq 1$. In [13, Lemma 5] we constructed, in a similar was as in Lemma 4.4, all numbers of the form $\frac{1+\sqrt{d}}{2}, d \in \mathbb{N}, d \equiv 1(\bmod 4)$, with all $a_{i}=1$ in symmetric part of the period. We have shown that all such numbers are of the form $\frac{1+\sqrt{d_{k}^{\prime}(n)}}{2}$, where $d_{k}^{\prime}(n)=4\left(\left(n \cdot F_{k}+1\right)^{2}+n \cdot F_{k-3}\right)+1, k, n \in \mathbb{N}$ or $2 n \in \mathbb{N}$ when $3 \mid k$. Some of those numbers was also given in [11, Example 5] $D_{k}(n)=4 F_{2 k}^{2} n^{2}+\left(20 F_{k}^{2}+8(-1)^{k}\right) n+5$, i.e., it is not hard to show that it holds $D_{k}(n)=d_{2 k}^{\prime}(n)$. It turns out that for the numbers in Lemma 4.4 it holds $d_{k}(n)=\frac{1}{4} d_{k}^{\prime}\left(\frac{2 n-1}{2}\right)$, and when $3 \nmid k$, continued fraction of $\sqrt{d_{k}(n)}$ have desired form (and there is no other number with such period).

Proof of Theorem 1.2. By (1.3), we have to prove

$$
R_{0}^{(3 M-1)}=\frac{p_{M \ell-2}}{q_{M \ell-2}}, \quad R_{0}^{(3 M)}=\frac{p_{M \ell-1}}{q_{M \ell-1}}, \quad R_{0}^{(3 M+1)}=\frac{p_{M \ell}}{q_{M \ell}} .
$$

We have $a_{0}=\frac{F_{\ell-3} F_{\ell}+1}{2}$, and since $3 \nmid \ell, F_{\ell-3}$ is odd, thus by Lemma 4.4 it holds

$$
\sqrt{d_{\ell}}=\left[a_{0}, \overline{\underbrace{1,1, \ldots, 1,1}_{\ell-1 \text { times }}, 2 a_{0}}\right] .
$$

From Cassini's identity, since $\ell$ is odd $(\ell \equiv \pm 1(\bmod 6))$, it follows

$$
\begin{aligned}
2 a_{0} & =F_{\ell-3}\left(F_{\ell-1}+F_{\ell-2}\right)+1=F_{\ell-2}^{2}+F_{\ell-3} F_{\ell-2}=F_{\ell-1} F_{\ell-2}, \\
d-a_{0}^{2} & =F_{\ell-3} F_{\ell-1}+1=F_{\ell-2}^{2} .
\end{aligned}
$$


So we get

$$
\begin{aligned}
R_{0}^{(1)} & =\frac{p_{0}}{q_{0}}=a_{0}, \\
R_{0}^{(2)} & =\frac{p_{0}^{2}+d q_{0}^{2}}{2 p_{0} q_{0}}=\frac{a_{0}^{2}+d}{2 a_{0}}=a_{0}+\frac{d-a_{0}^{2}}{2 a_{0}}=a_{0}+\frac{F_{\ell-2}}{F_{\ell-1}}=\frac{p_{\ell-2}}{q_{\ell-2}}, \\
R_{0}^{(3)} & =\frac{p_{0}\left(p_{0}^{2}+3 d q_{0}^{2}\right)}{q_{0}\left(3 p_{0}^{2}+d q_{0}^{2}\right)}=\frac{a_{0}\left(a_{0}^{2}+3 d\right)}{3 a_{0}^{2}+d}=a_{0}+\frac{2 a_{0}\left(d-a_{0}^{2}\right)}{4 a_{0}^{2}+d-a_{0}^{2}} \\
& =a_{0}+\frac{F_{\ell-1} F_{\ell-2}^{3}}{F_{\ell-1}^{2} F_{\ell-2}^{2}+F_{\ell-2}^{2}}=a_{0}+\frac{F_{\ell-1} F_{\ell-2}}{F_{\ell-1}^{2}+1}=a_{0}+\frac{F_{\ell-1} F_{\ell-2}}{F_{\ell-2} F_{\ell}} \\
& =a_{0}+\frac{F_{\ell-1}}{F_{\ell}}=\frac{p_{\ell-1}}{q_{\ell-1}} .
\end{aligned}
$$

Let us prove the theorem using induction on $M$. For proving the inductive step, first observe that from (2.6) for $m \geq 3$ we have

$$
R_{k}^{(m)}=\frac{R_{k}^{(2)} R_{k}^{(m-2)}+d}{R_{k}^{(2)}+R_{k}^{(m-2)}}, \quad R_{k}^{(m)}=\frac{R_{k}^{(3)} R_{k}^{(m-3)}+d}{R_{k}^{(3)}+R_{k}^{(m-3)}} .
$$

Suppose that for some $i \in\{0, \ell-2, \ell-1\}$ it holds $\frac{p_{(M-1) \ell+i}}{q_{(M-1) \ell+i}}=R_{0}^{(m-3)}$. We have

$$
\begin{aligned}
\frac{p_{M \ell+i}}{q_{M \ell+i}} & =[a_{0}, \underbrace{1,1, \ldots, 1,1}_{\ell-1 \text { times }}, a_{0}+\frac{p_{(M-1) \ell+i}}{q_{(M-1) \ell+i}}] \\
& =[a_{0}, \underbrace{1,1, \ldots, 1,1}_{\ell-1 \text { times }}, a_{0}+R_{0}^{(m-3)}] \\
& =\frac{p_{\ell-1}\left(a_{0}+R_{0}^{(m-3)}\right)+p_{\ell-2}}{q_{\ell-1}\left(a_{0}+R_{0}^{(m-3)}\right)+q_{\ell-2}} \stackrel{(3.4)}{=} \frac{p_{\ell-1} R_{0}^{(m-3)}+d q_{\ell-1}}{q_{\ell-1} R_{0}^{(m-3)}+p_{\ell-1}} \\
& \stackrel{(4.6)}{=} \frac{R_{0}^{(3)} R_{0}^{(m-3)}+d}{R_{0}^{(3)}+R_{0}^{(m-3)}} \stackrel{(4.7)}{=} R_{0}^{(m)} .
\end{aligned}
$$

Corollary 4.6. Let $\ell(\sqrt{d})=\ell$ be the length of the shortest period of the continued fraction expansion of $\sqrt{d}$. Then for each $m \geq 2$ it holds

$$
\sup _{d, n}\left\{\left|j_{n}^{(m)}(\sqrt{d})\right|\right\}=+\infty, \quad \limsup _{d, n}\left\{\frac{\left|j_{n}^{(m)}(\sqrt{d})\right|}{\ell(\sqrt{d})}\right\} \geq \frac{m}{6} .
$$




\section{Number OF GOOD APPROXIMANTS}

Analogously as in [2], let us define

$$
b^{(m)}(\alpha)=\mid\left\{n: 0 \leq n \leq \ell-1, R_{n}^{(m)} \text { is a convergent of } \alpha\right\} \mid .
$$

For arbitrary $m$ experimental results suggest that similar properties could hold as for $m=2$. However, there are some differences, as the following example shows.

EXAMPLE 5.1. We have $\ell(\sqrt{45})=6$ and

$$
b^{(m)}(\sqrt{45})=\left\{\begin{array}{lll}
4, & \text { if } m \equiv 2 \quad(\bmod 4), \\
6, & \text { if } m \neq 2 \quad(\bmod 4) .
\end{array}\right.
$$

Proof. We have $\sqrt{45}=[6, \overline{1,2,2,2,1,12}]=[\overline{6,1,2,2,2,1,6,0}]$. We will denote convergents of regular expansion with $\frac{p_{n}}{q_{n}}$. Using (2.3) and (2.4), $R_{2}^{(m)}=\frac{p_{3 m-1}}{q_{3 m-1}}$ and $R_{5}^{(m)}=\frac{p_{6 m-1}}{q_{6 m-1}}$ are good approximants, and by Theorem 1.1, we only have to check $R_{0}^{(m)}$ and $R_{1}^{(m)}$. The first few convergents of $\sqrt{45}$ are sequentially $\frac{6}{1}, \frac{7}{1}, \frac{30}{3}, \frac{47}{7}, \frac{114}{17}, \frac{161}{24}$, and let us observe how other convergents look like $\left([\ldots]_{M}\right.$ denote matrix form of convergents: $\left[a_{0}, a_{1}, \ldots, a_{n}\right]_{M}=$ $\left.\left(\begin{array}{cc}a_{0} & 1 \\ 1 & 0\end{array}\right)\left(\begin{array}{cc}a_{1} & 1 \\ 1 & 0\end{array}\right) \cdots\left(\begin{array}{cc}a_{n} & 1 \\ 1 & 0\end{array}\right)=\left(\begin{array}{cc}p_{n} & p_{n-1} \\ q_{n} & q_{n-1}\end{array}\right)\right)$, and let us write $\left(\frac{7+\sqrt{45}}{2}\right)^{3 k}=\gamma$.

$$
\begin{aligned}
\left(\begin{array}{ll}
p_{6 k+1} & p_{6 k} \\
q_{6 k+1} & q_{6 k}
\end{array}\right) & =[6,1,2,2,2,1,6,0]_{M}^{k}[6,1]_{M}=\left(\begin{array}{cc}
161 & 1080 \\
24 & 161
\end{array}\right)^{k}\left(\begin{array}{ll}
7 & 6 \\
1 & 1
\end{array}\right) \\
& =\left(\begin{array}{cc}
\gamma \frac{7+\sqrt{45}}{2}+\gamma^{\prime} \frac{7-\sqrt{45}}{2} & \gamma \frac{6+\sqrt{45}}{2}+\gamma^{\prime} \frac{6-\sqrt{45}}{2} \\
\frac{\gamma \frac{7+\sqrt{45}}{2}-\gamma^{\prime} \frac{7-\sqrt{45}}{2}}{\sqrt{45}} & \frac{\gamma \frac{6+\sqrt{45}}{2}-\gamma^{\prime} \frac{6-\sqrt{45}}{2}}{\sqrt{45}}
\end{array}\right), \\
\left(\begin{array}{ll}
p_{6 k+3} & p_{6 k+2} \\
q_{6 k+3} & q_{6 k+2}
\end{array}\right) & =\left(\begin{array}{ll}
\gamma \frac{47+7 \sqrt{45}}{2}+\gamma^{\prime} \frac{47-7 \sqrt{45}}{2} & \gamma \frac{20+3 \sqrt{45}}{2}+\gamma^{\prime} \frac{20-3 \sqrt{45}}{2} \\
\frac{\gamma \frac{47+7 \sqrt{45}}{2}-\gamma^{\prime}}{\sqrt{45}} & \frac{\gamma \frac{47-7 \sqrt{45}}{2}}{2}-\gamma^{\prime} \frac{20-3 \sqrt{45}}{2}
\end{array}\right), \\
\left(\begin{array}{ll}
p_{6 k+5} & p_{6 k+4} \\
q_{6 k+5} & q_{6 k+4}
\end{array}\right) & =\left(\begin{array}{cc}
\gamma \frac{161+24 \sqrt{45}}{2}+\gamma^{\prime} \frac{161-24 \sqrt{45}}{2} & \gamma \frac{114+17 \sqrt{45}}{2}+\gamma^{\prime} \frac{114-17 \sqrt{45}}{2} \\
\frac{\gamma \frac{161+24 \sqrt{45}}{2}-\gamma^{\prime} \frac{161-24 \sqrt{45}}{2}}{\sqrt{45}} & \frac{\gamma \frac{114+17 \sqrt{45}}{2}-\gamma^{\prime} \frac{114-17 \sqrt{45}}{2}}{\sqrt{45}}
\end{array}\right) .
\end{aligned}
$$

From (2.5) we have $R_{n}^{(m)}=\frac{\left(p_{n}+q_{n} \sqrt{45}\right)^{m}+\left(p_{n}-q_{n} \sqrt{45}\right)^{m}}{\left(p_{n}+q_{n} \sqrt{45}\right)^{m}-\left(p_{n}-q_{n} \sqrt{45}\right)^{m}} \sqrt{45}$. We see now that $R_{1}^{(m)}=\frac{(7+\sqrt{45})^{m}+(7-\sqrt{45})^{m}}{(7+\sqrt{45})^{m}-(7-\sqrt{45})^{m}} \sqrt{45}$ is always a good approximant. Namely, from $\left(\frac{7 \pm \sqrt{45}}{2}\right)^{2}=\frac{47 \pm 7 \sqrt{45}}{2}$ and $\left(\frac{7 \pm \sqrt{45}}{2}\right)^{3}=161 \pm 24 \sqrt{45}$, since $\frac{7}{1}, \frac{47}{7}$ and $\frac{161}{24}$ are convergents of $\sqrt{45}$, we have $R_{1}^{(m)}=\frac{p_{2 m-1}}{q_{2 m-1}}$. 
Finally, let us see when $R_{0}^{(m)}=\frac{(6+\sqrt{45})^{m}+(6-\sqrt{45})^{m}}{(6+\sqrt{45})^{m}-(6-\sqrt{45})^{m}} \sqrt{45}$ is a convergent. First consider

$$
\left(\frac{6 \pm \sqrt{45}}{3}\right)^{4}=161 \pm 24 \sqrt{45}=\left(\frac{7 \pm \sqrt{45}}{2}\right)^{3}
$$

From (5.1) we see $R_{0}^{(4 m)}=\frac{p_{6 m-1}}{q_{6 m-1}}$ and $R_{0}^{(4 m+1)}=\frac{p_{6 m}}{q_{6 m}}$, and since $(6 \pm \sqrt{45})^{3}=$ $9(114 \pm 17 \sqrt{45})$ and $\frac{114}{17}$ is a convergent of $\sqrt{45}$, we have $R_{0}^{(4 m+3)}=\frac{p_{6 m+4}}{q_{6 m+4}}$. From $(6 \pm \sqrt{45})^{2}=3(27 \pm 4 \sqrt{45})$, and since $\frac{27}{4}$ is not a convergent of $\sqrt{45}$, neither $R_{0}^{(4 m+2)}$ will be a convergent.

Let us define $\ell_{b}^{(m)}=\min \left\{\ell:\right.$ there exists $d \in \mathbb{N}$ such that $\ell(\sqrt{d})=\ell$ and $\left.b^{(m)}(\sqrt{d})=b\right\}$.

In [3] Dujella and the author proved that $\sup \left\{\frac{\ell_{b}^{(2)}}{b}: b \geq 1\right\} \leq 2$, and in

\begin{tabular}{|c|c|c|l||c|c|c|l|}
\hline$b$ & $\ell_{b}^{(3)} \leq$ & $d$ & $\ell_{b}^{(3)} / b \leq$ & $b$ & $\ell_{b}^{(3)} \leq$ & $d$ & $\ell_{b}^{(3)} / b \leq$ \\
\hline 3 & 5 & 13 & 1.666667 & 18 & 36 & 30420 & 2.941176 \\
4 & 6 & 21 & 1.5 & 19 & 71 & 313157 & 3.736842 \\
5 & 11 & 1625 & 2.2 & 20 & 44 & 193648 & 2.2 \\
6 & 6 & 45 & 1.0 & 21 & 41 & 21125 & 1.952381 \\
7 & 11 & 36125 & 1.571429 & 22 & 46 & 796500 & 2.0909091 \\
8 & 12 & 558900 & 1.5 & 23 & 157 & 221425 & 6.826087 \\
9 & 21 & 277 & 2.333333 & 24 & 66 & 740880 & 2.75 \\
10 & 14 & 500 & 1.4 & 25 & 97 & 490625 & 3.88 \\
11 & 37 & 828325 & 3.363636 & 26 & 50 & 29403 & 1.923077 \\
12 & 20 & 2548 & 1.666667 & 27 & 113 & 460525 & 4.185185 \\
13 & 45 & 74698 & 3.461538 & 28 & 78 & 84500 & 2.785714 \\
14 & 28 & 10125 & 2.461538 & 29 & 171 & 535517 & 5.896552 \\
15 & 41 & 9125 & 2.733333 & 30 & 80 & 41405 & 2.666667 \\
16 & 28 & 1125 & 1.75 & 31 & 97 & 903125 & 3.129032 \\
17 & 67 & 260389 & 3.941176 & 32 & 88 & 892125 & 2.75 \\
\hline
\end{tabular}

TABLE 1. Upper bounds for $\ell_{b}^{(3)}, 3 \leq b \leq 32$.

[13] the author showed the same inequality for $\alpha=\frac{1+\sqrt{d}}{2}, d \in \mathbb{N}, d \equiv 1$ $(\bmod 4)$ and $d$ is not a perfect square. In Table 1 we show upper bounds for $\ell_{b}^{(3)}$, obtained by experiments, and corresponding $d$ 's (we tested all $d$ 's smaller then $10^{6}$ ). Other experiments (we tested all $m$ 's until 20) give similar upper bounds, but $b^{(m)}(\sqrt{d})$ is not a monotonic function in $m$. Experimental results lead to the conclusion that for every positive integer $m \geq 3$ and every positive 
integer $b$ there exist a positive integer $d$ such that $b^{(m)}(\sqrt{d})=b$. Moreover, obtained upper bounds for $\frac{\ell_{b}^{(m)}}{b}$ suggest that

$$
\sup \left\{\frac{\ell_{b}^{(m)}}{b}: b \geq 1\right\} \leq 2
$$

for all $m \geq 2$. In case $m=2$ families of examples where constructed which show that for every positive integer $b$ there exist a positive integer $d$ such that $b^{(2)}(\sqrt{d})=b$ and $b^{(m)}(\sqrt{d})>\ell(\sqrt{d}) / 2$. To prove the inequality (5.2) for each $m \geq 3$ in a similar manner seems nearly impossible because $b^{(m)}(\sqrt{d})$ depends not only on $d$ but also on $m$ (see Example 5.1).

ACKNOWLEDGEMENTS.

The author is grateful to Andrej Dujella and the referee for many useful comments and remarks on the previous version of this paper.

\section{REFERENCES}

[1] G. Chrystal, Algebra, Part II, Chelsea, New York, 1964.

[2] A. Dujella, Newton's formula and continued fraction expansion of $\sqrt{d}$, Experiment. Math. 10 (2001), 125-131.

[3] A. Dujella and V. Petričević, Square roots with many good approximants, Integers 5(3) (2005), \#A6. (electronic)

[4] N. Elezović, A note on continued fractions of quadratic irrationals, Math. Commun. 2 (1997), 27-33.

[5] E. Frank, On continued fraction expansions for binomial quadratic surds, Numer. Math. 4 (1962), 85-95.

[6] E. Frank and A. Sharma, Continued fraction expansions and iterations of Newton's formula, J. Reine Angew. Math. 219 (1965), 62-66.

[7] A. S. Householder, The Numerical Treatment of a Single Nonlinear Equation, McGraw-Hill, New York, 1970.

[8] T. Komatsu, Continued fractions and Newton's approximants, Math. Commun. 4 (1999), 167-176.

[9] J. Mikusiński, Sur la méthode d'approximation de Newton, Ann. Polon. Math. 1 (1954), 184-194.

[10] R. A. Mollin, Infinite Families of Pellian Polynomials and their Continued Fraction Expansions, Results Math. 43 (2003), 300-317.

[11] R. A. Mollin and K. Cheng, Continued Fraction Beepers and Fibonacci Numbers, C. R. Math. Rep. Acad. Sci. Canada 24 (2002), 102-108.

[12] O. Perron, Die Lehre von den Kettenbrüchen I, Dritte ed., B. G. Teubner Verlagsgesellschaft m.b.H., Stuttgart, 1954.

[13] V. Petričević, Newton's approximants and continued fraction expansion of $\frac{1+\sqrt{d}}{2}$, Math. Commun. 17 (2012), 389-409.

[14] P. Sebah and X. Gourdon, Newton's method and high order iterations, preprint, 2001. http://numbers. computation.free.fr/Constants/Algorithms/newton.ps

[15] A. Sharma, On Newton's method of approximation, Ann. Polon. Math. 6 (1959), $295-300$.

[16] K. S. Williams and N. Buck, Comparision of the lengths of the continued fractions of $\sqrt{D}$ and $\frac{1+\sqrt{D}}{2}$, Proc. Amer. Math. Soc. 120 (1994), 995-1002. 
V. Petričević

Department of Mathematics

University of Zagreb

10000 Zagreb

Croatia

E-mail: vpetrice@math.hr

Received: 6.9.2012.

Revised: 5.11.2012. 